\title{
Assessment of cracks in lateral supports of the magnet system of Wendelstein 7-X
}

\author{
Joris Fellinger $^{\mathrm{a}}$, Victor Bykov ${ }^{\mathrm{a}}$, Felix Schauer ${ }^{\mathrm{a}}$ \\ ${ }^{a}$ Max Planck Institute for Plasma Physics, EURATOM Association, Wendelsteinstr. 1, 17491 Greifswald, Germany
}

\begin{abstract}
The superconducting coils of the magnet system of Wendelstein 7-X (W7-X) are bolted onto a central support ring and interconnected with five so-called lateral support elements (LSEs) per half module. After welding of the LSE hollow boxes to the coil cases cracks were found in the vicinity of the welds that could potentially limit the allowed number $N$ of electromagnetic (EM) load cycles of the machine.

In response to the appearance of first cracks during assembly, the stress intensity factor (SIF) of theoretical cracks of various sizes in potentially critical position and orientation were predicted in a fast approach. For each crack size, $N$ was based on the SIF, derived from beam theory, and on Paris' law parameters determined in fatigue crack growth rate (FCGR) tests, thus leading to tolerable maximal crack sizes and distances between cracks. It was proved that the actual crack dimensions remained below these values or turned out to be only superficial. Afterwards, (extended) finite element method (XFEM and FEM) and boundary element method (BEM) models were developed to project the SIF of most critical tolerated cracks, considering new FCGR tests and the local stress state in more detail. $N$ appeared highly sensitive to the assumptions which were therefore critically reviewed.

Finally, the limit for load combinations of different amplitudes was determined using Miner's rule. As a result it was shown that the predefined number of W7-X operation cycles is not jeopardized by any of the detected cracks.
\end{abstract}

Keywords: crack, stress intensity factor, Paris' law, XFEM, BEM, W7-X, magnet system.

\section{Introduction}

The modular stellarator $\mathrm{W} 7-\mathrm{X}$ is currently under construction in Greifswald, Germany. The magnet system consists of 50 non-planar (NP) and 20 planar superconducting coils to be operated at $4 \mathrm{~K}$. The NP coil cases are made of cast stainless steel (SS) EN 1.3960. They are bolted onto a SS central support ring and welded together at the outboard side of the torus via the LSE's consisting of $100-150 \mathrm{~mm}$ long hollow "beams" of $30-35 \mathrm{~mm}$ thick forged SS EN 1.4429 (s. figure 1). After welding the LSEs (weld depth 15-30 mm), many surface cracks substantially larger than $8 \mathrm{~mm}$ (typical acceptance limit of EN 23277 [1]) were found with dye penetration tests at the accessible surfaces, particularly at the coil side of the weld within the cast steel.

To avoid a reduction of $N$ due to unstable crack growth, the crack sizes and spans that could be tolerated without repair was determined based on the prediction of crack propagation during operation.

First, a fast approach was developed to predict the SIF around hypothetical cracks of various sizes in potentially critical position and orientation (see Section 2). For each crack size, $N$ was predicted based on Paris' law parameters fitted on FCGR test data and on the calculated SIF, thus leading to tolerable crack sizes.

Afterwards, detailed FEM and BEM models of actually tolerated cracks in the LSEs were developed to project the SIF and the corresponding crack propagation (see Section 3). In the FEM models the crack was either modeled as an unconnected seam between adjacent elements with a crack following mesh (seam technique) or using the XFEM technique with a crack-independent mesh. The XFEM technique appeared to be very meshdependent. The seam technique was successfully benchmarked against a BEM model and analytical results. So, it formed the basis of the final assessment.

In Section 4, the different techniques are compared. $N$ appears highly sensitive to the assumptions of the assessment. The detailed models include the effect of stresses around the cracks imposed by coil deformation. Moreover, new FCGR tests led to updated Paris' law parameters which also significantly affected the final prediction of $N$.

The envelope of allowed load cycle combinations of different amplitude and mean value is presented for the most critical cracks in Section 5. The envelope is constructed using Miner's rule. It is confirmed that the specified number of operational cycles is not jeopardized by any of the observed cracks.

\section{Fast approach}

During assembly, a tool was required for rapid decisions whether cracks observed in dye penetration tests required repair. Therefore a fast analytical approach was developed demonstrating that theoretical semicircular cracks in the most critical locations with initial radius $a_{i}$ of $5 \mathrm{~mm}$ (in the weld) or $10 \mathrm{~mm}$ (in the cast material) did not limit $N$ to less than the specified load cycles for operation of W7-X [2]. The procedure reads

1: The cracks are parallel to the weld seam in eight positions on both sides along the outside of the hollow LSE cross section in both the weld and in the cast steel, midway between the weld and the coil case, see figure 1 . 


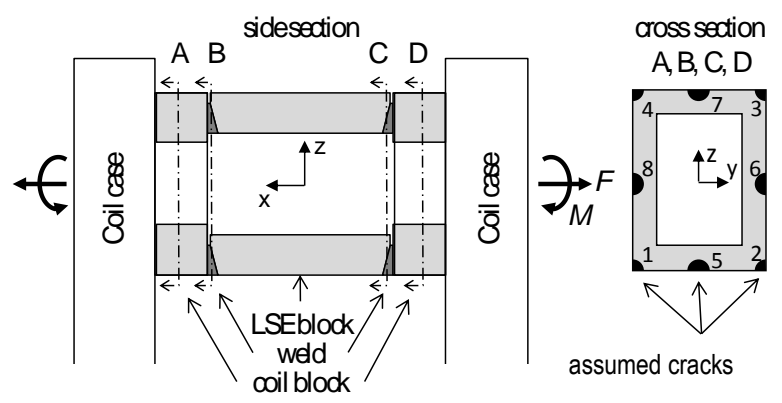

Fig.1 Cross sections and positions of theoretical cracks

2: Based on forces and moments extracted from global FEM models for nine default EM configurations [3], the normal stresses perpendicular to the crack and the shear stresses in the crack plane are calculated for each of the $8 \times 4$ potential crack positions based on simple beam theory. The reduction of the cross section by the crack is not taken into account. As a first approximation the average normal stress over the thickness of the tubular shape was taken. Later on, the higher normal stress at the edge was used.

3: The SIF for different crack modes $K_{I}, K_{I I}, K_{I I}$ are calculated along the crack front according to [4] based on stress components of step 2. The final SIF $K_{e q \alpha}$ that was used to predict crack growth under an arbitrary angle $\alpha$ with the initial crack plane is defined as

$$
K_{e q \alpha}=\sqrt[4]{K_{e q}{ }^{4}+4 K_{I}^{2} K_{I I}^{2}}
$$

in which

$$
K_{e q}=k \sqrt{K_{I}^{2}+K_{I I}^{2}+\frac{1}{1-v} K_{I I I}^{2}}
$$

where $k$ is a correction factor to account for finite thickness of the tubular cross section according to [5].

4: The crack growth is predicted using Paris' law

$$
\frac{d a}{d N}=C d K^{m}
$$

with material parameters $C$ and $m$ derived from FCGR test series carried out under cryogenic temperatures at KIT, Karlsruhe, see figure 2. The original assessment used the fit curve 1 in figure 2, based on a single test. Later on, tests were grouped depending on the test series and type of material through which the crack actually grew during the test, i.e. through the weld material, the heat affected zone (HAZ) or the base materials (either EN 1.3960 or EN 1.4429). Since parameter $m$ (slopes in figure 2) was typically higher than the expected literature values of around 3-4, see for instance [6], extra tests were carried out on cast material for $15 \leq K_{I} \leq 70 \mathrm{MPa} \sqrt{\mathrm{m}}$ showing that the steep slope of the green tests are most likely caused by the fact that the crack initiation phase was predominantly measured rather than the stable crack growth phase, thus leading to too conservative Paris' law parameters. Fit curve $2 \mathrm{c}$ therefore neglects the initial measurements (presented without the circles around the dots in figure 2). Finally, for cracks in welds and the cast steel the fit curves $2 \mathrm{w}$ and $2 \mathrm{c}$, respectively were used.

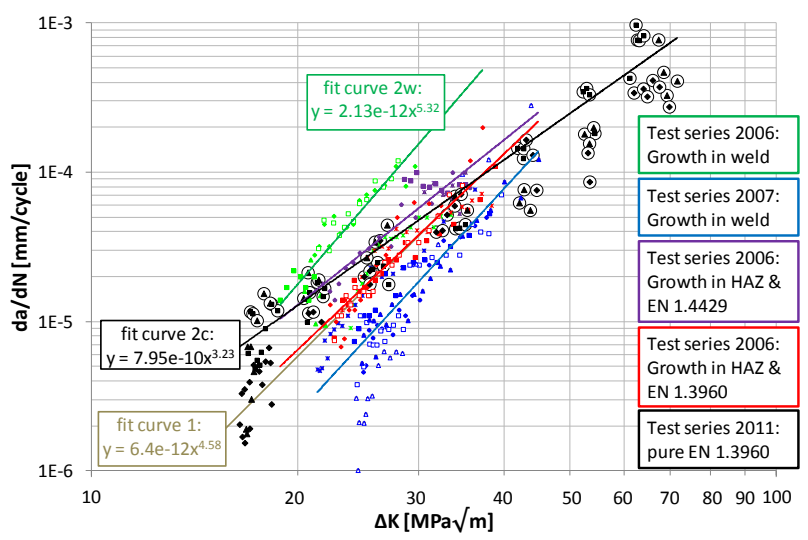

Fig.2 FCGR test results of welded and not welded stainless steels at $7 \mathrm{~K}$. (HAZ $=$ heat affected zone)

5: The crack growth was limited to the ultimate radius $a_{u}$ defined by either (1) reaching the critical crack intensity, (2) reaching the crack size for which the elastic approach of [4] and [5] is no longer valid, or (3) twice $a_{i}$. The critical stress intensity was conservatively taken as $2 / 3$ times the lowest value of the critical stress intensity as measured in fracture toughness tests. The elastic approach is considered to be justified as long as the radius of the plastic zone at the crack tip is small in comparison with the radius of the K-dominated stress zone. According to [7] this requirement is fulfilled if:

$$
a \geq 2.5\left(\frac{K}{f_{y}}\right)^{2}
$$

with $a$ the crack radius and $f_{y}$ the yield limit of the base material. $K_{e q \alpha}$ was used for $K$. The third limit mentioned above was set to prevent adjacent cracks to coalesce during crack growth and to justify the calculation of the stresses using the un-cracked cross sectional properties.

6: $N$ was calculated using $K \propto \sigma \sqrt{a}$ and assuming constant remote stress level during crack growth. Thus:

$$
\Delta K=\frac{\sqrt{a}}{\sqrt{a_{i}}} \Delta K_{i},
$$

where $K_{i}$ is the SIF at the initial crack size $a_{i} . N$ is obtained from eq. (3) and (5) using:

$$
N=\int_{a_{i}}^{a_{u}} \frac{1}{C \Delta K^{m}} d a=\frac{1}{C}\left(\frac{\sqrt{a_{i}}}{\Delta K_{i}}\right)^{m} \int_{a_{i}}^{a_{u}} a^{-m / 2} d a=\frac{A}{C} \Delta K_{i}^{-m}
$$

with

$$
A=a_{i}^{m / 2} \frac{a_{u}^{(1-m / 2)}-a_{i}^{(1-m / 2)}}{1-m / 2} .
$$

Finally, $N$ was calculated for each of 32 theoretical cracks in five LSE types under loads for all nine default EM configurations defined for $\mathrm{W} 7-\mathrm{X}$ at $2.5 \mathrm{~T}$ and $3 \mathrm{~T}$ operation regimes.

Changing the Paris' law parameters from fit curve 1 to $2 \mathrm{w}$ leads to a reduction of $N$ by a factor 5 . Using the maximum normal stress over the thickness of the block instead of the mean value leads to 1.2-1.4 times larger normal stress and SIF. From equation (6) it can be 
directly obtained that this leads to a further reduction of $N$ by a factor 2-5 depending on $m$.

\section{XFEM, FEM and BEM modeling}

As explained in the previous section, $N$ is sensitive to the stress. So, accepted cracks were feared to become critical due to local stress induced by coil deformation, contact at the not fully penetrated weld and reduction of cross section by the crack.

First, the XFEM technique was used in FEM code ABAQUS v6.11-1. With the XFEM method a crack of any shape can be easily introduced in any position and orientation without adapting the original mesh. $K$ is derived from the mean J-integral along the crack tip as:

$$
K=\sqrt{\frac{E}{1-v^{2}} J}
$$

$N$ was then calculated using equation (6). However, several problems appeared: The J-integral became sometimes negative for cracks subjected to compressive stress which is theoretically impossible. Thermal strains modified the result dramatically even if a stress free thermal strain field was applied, and most importantly, $K$ appeared to be too sensitive to the mesh: For a $2 \%$ increase of the crack size with the same mesh, up to $50 \%$ decrease of $K$ was calculated instead of an increase.

Therefore, the cracks were modeled once again with the seam technique, see figure 3, to overcome all XFEM related problems.

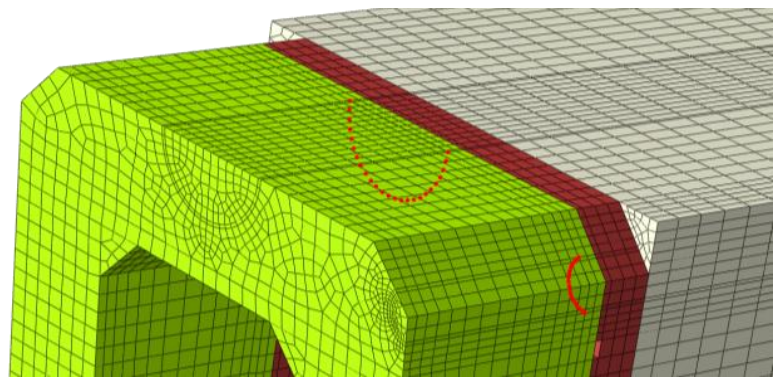

Fig.3 Mesh with highlighted cracks modeled as seams

Each LSE was modeled in detail including shape of the weld and the contact between LSE block and coil case block. In order to evaluate the effect of coil deformation, the sub-modeling technique was used, i.e. the displacements at the cuts towards the coils were taken from the ABAQUS global model (GM) of the magnet system [3] as boundary conditions.

To verify the FEM model with seams and the assumption that the crack growth can be estimated by the mean J-integral along the crack front, LTCalcoli in Italy performed a validation for one crack using the BEM method. This allows for a prediction of non-uniform 3D crack growth along the crack front which is determined by Paris' law parameters and the calculated local SIF. The BEM model was made in BEM code BEASY and loaded with the GM displacements.

\section{Discussion}

The mean SIF of the BEM model was within $10 \%$ in comparison with the seam technique. The SIF along the crack front varies only within $\pm 7 \%$ during all stages of crack growth, see BEM results in figure 4, justifying averaging the SIF along the front to predict uniform crack growth. Moreover, peak values of the SIF do not coincide with largest local crack radius, and the position of the peak changes during crack growth, see figure 4 .
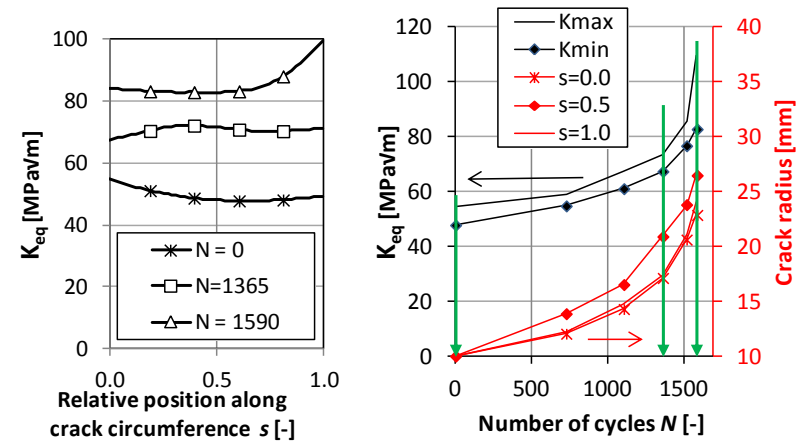

Fig.4 SIF along crack front s during growth (left) and SIF and crack size change with cycles (right)

With the seam technique the effect of the contact without weld penetration between the LSE and coil block was investigated. If a crack is next to a contact (top wall of LSE in figure 1), the contact 'shields' the crack from the normal stress and the SIF is reduced. If the crack is at the opposite side of the wall as the contact (bottom wall of LSE in figure 1), the contact increases the normal stresses at the crack. The effect depends on the distance between the weld and the crack. For a crack in the middle of the coil block, the SIF is increased by some $5 \%$ only.

The effect of the stresses due to the coil case deformation on the SIF cannot be neglected. Figure 5 shows the differences comparing a LSE once loaded with the coil displacements of the GM (submodel technique) and once with the forces and moments extracted from the same GM. Clearly, the stresses strongly increase towards the coil due to coil deformation causing an increase of the SIF up to $40 \%$. As mentioned before, such increase of the SIF reduces $N$ by a factor 5 , depending on the power in the Paris law.

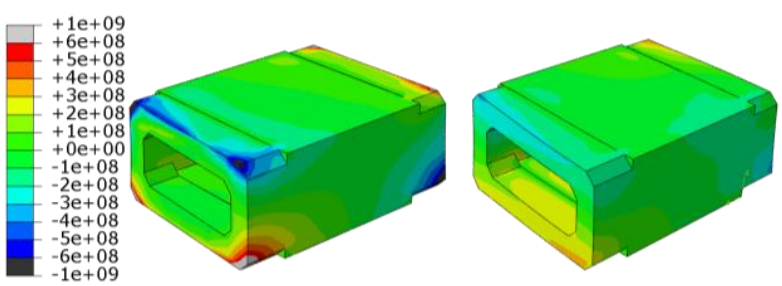

Fig. 5 Normal stress $\left(\mathrm{N} / \mathrm{m}^{2}\right)$ due to EML with (left) and without (right) local coil deformation

\section{Operation limits}

Considering the detailed stress states and updated Paris law parameters, 16 accepted cracks were identified as potentially critical. For these cracks the operational limits were evaluated in more detail.

It is planned that the magnet system will remain loaded for a 5-days week of experimental operation, with only a limited reduction of the EM field at nights. During an experimental period the EM field can be 
shifted several times from one plasma configuration to another, and also from $2.5 \mathrm{~T}$ to $3 \mathrm{~T}$ operation and back, see the scheme in figure 6 .

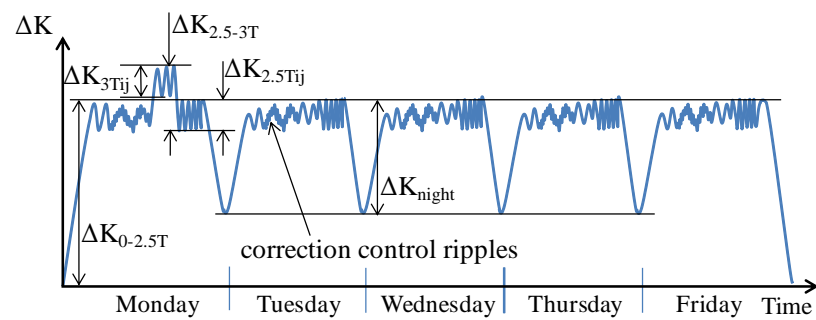

Fig.6 Schematic loading history due to EM forces

To estimate the operation limits, the following SIF increments had to be considered: $\Delta K_{0-2.5 T}$ due to start up of the machine on Monday morning from 0 to $2.5 \mathrm{~T}$, $\Delta K_{2.5-3 T}$ due to increase from $2.5 \mathrm{~T}$ to $3 \mathrm{~T}, \Delta K_{2.5 T i j}$ and $\Delta K_{3 T i j}$ due to each shift from one EM configuration to another at $2.5 \mathrm{~T}$ and $3 \mathrm{~T}$ respectively and $\Delta K_{\text {night }}$ due to the field reduction at night. Changes in the EM field for plasma control are small enough to be neglected. At first, the SIF at $2.5 \mathrm{~T}$ and $3 \mathrm{~T}$ were calculated for the nine specified EM configurations and for all 16 potentially critical cracks. From these results, the SIF increments were calculated for all possible shifts and the maxima were used as basis for the calculation of the corresponding $N_{i}$. For all load cycles except those from 0-2.5 T, Paris' law was modified to account for the nonzero initial value of $K$ over the increment according to $[7$, p. 454] as

$$
\frac{d a}{d N}=\frac{C^{\prime}}{K_{I c} / K_{\max }-1} \Delta K^{m^{\prime}}
$$

In which $K_{I c}$ is the critical stress intensity factor obtained in fracture toughness tests and $K_{\max }$ the maximum stress intensity in a load cycle. To account for the growth of $K_{\max }$ over the cycles, the value at ultimate crack size was used, i.e. $K_{\max , u}=K_{\max , u} \sqrt{a_{u} / a_{i}} \cdot N$ is calculated according to eq. (6), using $C^{\prime}$ and $m^{\prime}$ which were fitted against the FCGR test data using eq. (9) and

$$
A^{\prime}=\frac{K_{I c} / K_{u, \max }-1}{C^{\prime}} a_{i}^{m / 2} \frac{a_{u}^{\left(1-m^{\prime} / 2\right)}-a_{i}^{\left(1-m^{\prime} / 2\right)}}{1-m^{\prime} / 2}
$$

Assuming 4 times more field reductions at night than start-ups, $k_{1}$ times more $2.5 \mathrm{~T}$ cycles than $3 \mathrm{~T}$ cycles, and $k_{2}$ times more shifts from one EM configuration to another than from zero to nominal EM load (for both $2.5 \mathrm{~T}$ and $3 \mathrm{~T}$ ), the number of $2.5 \mathrm{~T}$ cycles $n$ were calculated using Miner's rule with the safety factor $\gamma$ equal to 10 as

$$
\frac{n}{N_{2.5 T}}+\frac{4 n}{N_{n i g h t}}+\frac{n}{k_{1} N_{2.5-3 T}}+\frac{k_{2} n}{N_{2.5 T i j}}+\frac{k_{2} n}{k_{1} N_{3 T i j}} \leq \frac{1}{\gamma}
$$

It could be shown that all cracks allow for at least one thousand $2.5 \mathrm{~T}$ cycles plus hundred $3 \mathrm{~T}$ cycles, and 20 switches per cycle from one EM configuration to another. However, an inevitable risk remains that some cracks were not detected because not all potential crack locations were accessible for dye penetration testing. Nevertheless, the selected approach is based on conservative assumptions and criteria:

- Crack growth limitation $a_{u}=20 \mathrm{~mm}$

- No unstable crack growth. At $a_{u}: K_{\max }<0.5 K_{I c}$

- Crack depth is equal to the crack radius, real cracks were found to be much more superficial

- Crack growth retardation due to crack tip yielding under high loads is not considered

- The largest $\Delta K$ over all nine EM configurations is taken for all loading situations

- The safety factor 10 is usually taken for analytical techniques, which do not necessarily include detailed effects like coil deformation.

- Finally, the consequences of a hypothetically fully cracked LSE were assessed. Such a worst case would only cause coil deformations which would be detrimental for plasma operation, but no progressive failure or collateral damage would occur.

\section{Conclusions}

Fast analytical and advanced FEM, XFEM and BEM methods to calculate the SIF around cracks in LSE were developed and successfully verified against each other.

Extensive FCGR tests on welded and cast SS were carried out showing wide scatter.

All cracks which formed during welding were acceptable according to the fast approach criterion. The seam technique in FEM confirmed that they do not limit the number of the planned operational cycles even though these FE analyses, taking into account local secondary stresses and conservative Paris' law parameters, resulted in considerably lower allowed cycle numbers.

\section{Acknowledgments}

The discussions with Prof. D. Gross (TU Darmstadt, Germany), Dr. M. Janssen (TU Delft, the Netherlands), Dr. A. Nyilas (CEME Stutensee, Germany), and Dr. K.P. Weiss (KIT Karlsruhe, Germany) are highly appreciated. The BEM modeling, carried out under responsibility of LTCalcoli (Merate, Italy) and the material tests, carried out by KIT (Karlruhe, Germany) are also kindly acknowledged. Special thanks go to Dr. L. Sonnerup who developed the fast approach with contributions from our colleagues D. Zacharias and K. Egorov.

\section{References}

[1] EN ISO 23277, (CEN Brussels 2010)

[2] L. Sonnerup, IPP internal report 1-GXA-T0092 (2008)

[3] V. Bykov et al., Fus. Eng. Des. 84, No. 2-6 (2009), pp 215

[4] D. P. Rooke and D. J. Cartwright, Compendium of Stress intensity factors, (HMSO, London 1976)

[5] J.C. Newman, I.S. Raju, Eng. Frac. M. 15, (1981), pp. 185

[6] N.A. Fleck, J.J. Kang, M.F. Asby, Acta metal. mater. 42, No.2, (1994), pp. 365-381

[7] T.L. Anderson, Fracture mech. (Taylor \& Francis 2005) 